\title{
Are happy residents better residents?
}

\author{
Marike L. Broekman ${ }^{1,2}$ • Saskia Peerdeman ${ }^{3,4}$
}

Received: 29 July 2016 / Accepted: 1 August 2016 / Published online: 17 August 2016

(C) Springer-Verlag Wien 2016

The paper by Stienen et al. shows that residents' satisfaction with the quality of theoretical training correlates with higher scores on the European Board Examination in Neurosurgery [3]. Even though it would have been interesting to examine what effect year of residency or English proficiency of those taking the exam have on the score, this study highlights some important points for training of our future colleagues.

The question arises as to whether the residents are more satisfied because they have developed greater knowledge, or whether their knowledge is greater as a result of a more satisfying training? Indeed, the data presented could indicate that those satisfied by their training are more intrinsically motivated, might be more motivated to studying, and will subsequently do better on tests. Intrinsic motivation has been defined as: "the doing of an activity for its inherent satisfaction rather than for some separable consequence" [2]. It is the result of satisfaction of three basic needs: having autonomy, feeling competent to perform tasks and having relatedness, and with support of significant others [1] and has been shown to correlate with deep learning, academic success, and positive well-be-

Marike L. Broekman

m.l.d.broekman-4@umcutrecht.nl

1 Department of Neurosurgery, Brain Center Rudolf Magnus, University Medical Center Utrecht, Utrecht, Netherlands

2 Department of Neurology, Massachusetts General Hospital, Harvard Medical School, Boston, MA, USA

3 Department of Neurosurgery, VU University Medical Center, Amsterdam, The Netherlands

4 VU University Medical Center School of Medical Sciences, Amsterdam, The Netherlands ing. Residency programs that respect the three basic needs necessary for intrinsic motivation will train happy residents that will perform better on tests.

However, it should be noted that the European Board Examination in Neurosurgery only tests for one aspect of neurosurgery: theoretical knowledge. Other competences, such as technical skills, non-technical skills (leadership, decisionmaking, team performance, time management), justified confidence, and attitude are not tested. Indeed, the test scores, including that of the second part of the exam, which aims to test more clinical decision-making/solving of clinical cases, did not correlate with residents' satisfaction with practical training.

Interestingly, the authors found no difference in performance on the theoretical test between men and women. As expected, intellectual capacity is not gender specific. The authors correctly point out that circumstances during residency are still not equal for both sexes. Whereas we do not think this might have a tremendous impact on their satisfaction with theoretical training, we think it will influence satisfaction with other aspects of residency.

The working time regulations have been subject of much debate. Even though many have argued that the regulations have a negative impact on residents' knowledge, this study clearly shows that working longer hours does not result in higher tests scores. This could indicate that residents who are well rested and satisfied will be more inclined to spend extra time studying, time that would otherwise not necessarily be spent on activities that foster learning, like administrative duties. It is important to realize that simply spending more hours in a hospital will not make for better neurosurgeons. However, using those hours to create an environment that fosters intrinsic motivation, for instance by theoretical teaching integrated in daily practice, will be an efficient way to train our future happy, skilled, and smart neurosurgeons. 


\section{References}

1. Dec EL, Ryan RM (2008) Self-determination theory: a macro theory of human motivation, development and health. Can Psychol 49:182185
2. Ryan RM, Deci EL (2000) Intrinsic and extrinsic motivations: classic definitions and new directions. Contemp Educ Psychol 25:54-67

3. Stienen MN, Netuka D, Demetriades AK, Ringel F, Gautschi OP, Gempt J, Kuhlen D, Schaller K (2016) Residency program trainee-satisfaction correlate with results of the European Board Examination in Neurosurgery. Acta Neurochir. doi:10.1007/s00701-016-2917-y 